\title{
A Rare Origin of Supraclavicular Mass in a Ten- Month-Old Child: Case Report of Lipoblastoma
}

\author{
Merzouqi B, Salama K*, Khdim M, Rouadi S, Abada A, Roubal M and Mahtar M \\ Department of ORL and Neck Surgery, University Hospital Center Ibn Rochd, Morocco
}

Submission: November 19, 2018; Published: December 13, 2018

*Corresponding author: Salama Khadija, ORL and Neck surgery Department, University Hospital Center Ibn Rochd, Casablanca, Morocco

Keywords: Lipoblastoma; Painless; Non-pulsatile; Hemangioma; Cervical mass; Subclavian vessels; Cleidotomy

\section{Introduction}

Lipoblastoma is a rare benign tumor arising from embryonic white fat which occurs in the early childhood and accounts for up to $30 \%$ of adipocytic tumors in children while true lipomas account for $60 \%$ of all cases [1]. It usually arises on the extremities and considered as a rare cause of a pediatric head and neck masses. To notice that less than 100 cases of lipoblastomas- all locations- have been reported in literature till now, whereas, head and neck involvement has been reported in only very few cases [2]. In the Hospital University Ibn Rochd, we admitted a ten month child with lipoblastoma arising from the supraclavicular area. A significant risk of invading the subclavian vessels, the brachial plexus and the thoracic duct in contact was near.

Case Report

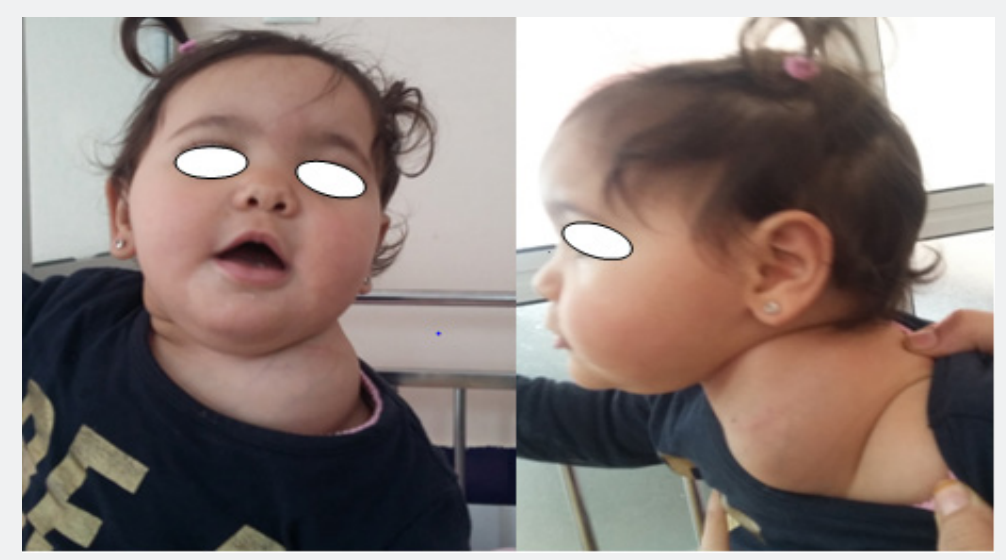

Figure 1: pre-operative image of the large supraclavicular mass.

A 10-month-old girl, with no relevant medical or surgical history, brought by her parents to the outpatient ENT department for painless left side neck mass growing progressively over the last 5 months, with no sign of compression, in a context of a conservation of general state. On physical examination, there was a painless, non-pulsatile, solid to firm left-sided cervical mass in the supraclavicular area measuring approximately $6 \mathrm{~cm}$ of large diameter, fixed to the underlying structures, mobile relative to the overlying skin (Figure 1), with no inflammatory signs neither was a lymph node hypertrophy. The rest of clinical examination was within normal limits with no abnormal laboratory findings. Ultrasonography revealed a homogeneous, hyperechogenic mass with a peripheral vascularization, plunging into the left supraclavicular area evocative of either hemangioma or lipoblastoma. We completed by neck CT scan which revealed a well limited supraclavicular lobulated mass encroaching upon the sternocleidomastoid muscle, with a greasy density similar to that of the subcutaneous fat in which some enhancement was visible, crossed by internal septa, measuring $63 \times 42 \times 40 \mathrm{~mm}$. The mass came into contact with the clavicle without bone lysis, also with the subclavian blood vessels which remain permeable (Figure 2). The biopsy of the mass confirmed the diagnosis of lipoblastoma. A multidisciplinary surgery (Otorhinolaryngologist and Pediatric surgeon) was considered. 
Per-operatively, the lesion was found to be well encapsulated fixed to clavicle, in contact with the branches of the brachial plexus posteriorly. Complete surgical resection of the mass with an under-periosteum dissection without cleidotomy was performed, the subclavian vein was tightly adherent to the mass which was delicately dissected, and the brachial plexus was seen and preserved (Figure 3). Histological examination showed an adipose lesion, divided into lobules by fibrous septa. The adipose tissue consisted of mature adipocytes of various size and few lipoblasts. These findings were compatible with lipoblastoma. The postoperative period was uneventful, one-year follow-up found no local recurrence.
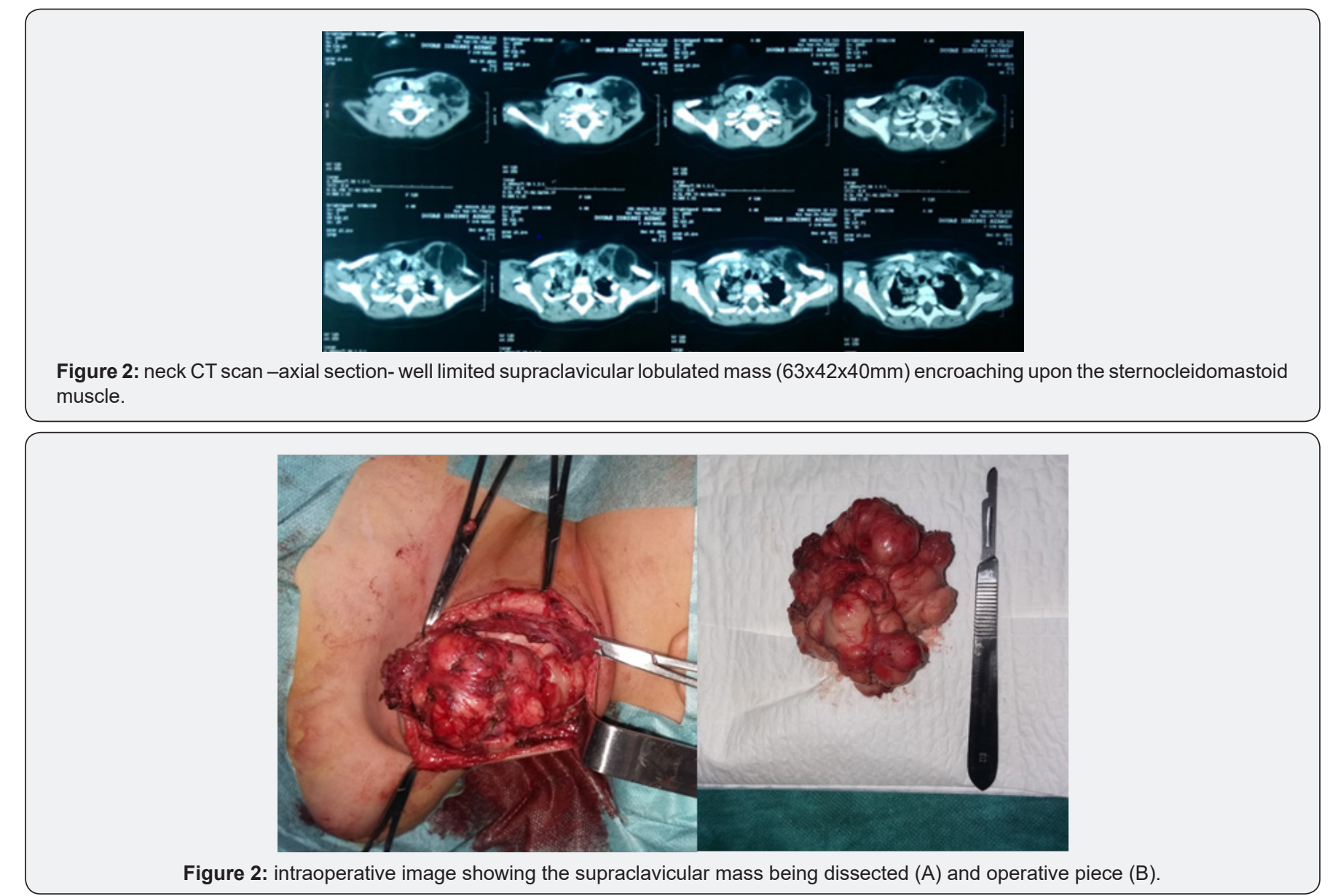

\section{Discussion}

The term "lipoblastoma" was coined by Jaffe [3] in 1926 to describe an atypical lipomatous lesion that consisted of cells resembling embryonic white fat [3]. This term was intended to differentiate these lesions from common lipomas, which contain no lipoblasts. Chung \& Enzinger [1] described two forms: lipoblastoma as a localized circumscribed type which represents approximately $70 \%$ of cases and lipoblastomatosis for the diffuse multicentric variant (about 30\% of cases) which is a deeply located, poorly circumscribed lesion with infiltrative growth pattern that may affect surrounding muscle structures, this latter is more difficult to remove completely $[1,4]$. It commonly arises from the extremities (36 72\%) and the trunk (20 50\%), and less commonly in the head and neck (10 15\%) [2]. Within this latter, the neck is the most common location, other locations may also be included as the parotid gland, cheek, skin and orbit $[5,6]$. It occurs exclusively in infants and children, with $90 \%$ of the cases in children under 3 years of age, with the median age of onset being 1 year. It shows a male preponderance (3:1) and a rapid growth rate [6].
Usually, it presents as a painless, progressively growing mass if localized superficially. Symptoms are related to the location and size or mass effect of the lesion. Its rapid growth may cause compressive symptoms such as an airway obstruction and respiratory symptoms that have been described in patients with pleural, mediastinal, pulmonary, and lower neck lipoblastomas $[5,7]$.

The presumptive diagnosis is performed by imaging: Ultrasonography, CT or MRI being more sensitive by showing the characteristics of the mass components [7]. Imaging exhibits the precise location of the lesion and its extent to the surrounding structures which helps in planning surgical resection, however, it cannot differentiate lipomatous tumors which make these ones radiologically indistinguishable [8]. Therefore, the definitive diagnosis is made histologically. Moreover, recent cytogenetic analysis revealed specific chromosomal abnormalities in adipose tissue tumors which may lead to accurate diagnosis [2,9].

The most important differential diagnosis of lipoblastoma is myxoid liposarcoma. Since the distinction between the two 
entities cannot be performed on imaging. The age of the patient is significant. Liposarcomas are extremely rare in patients less than 10 years of age. Histology can differentiate on the basis of architectural changes and cytogenetic analysis displays a characteristic rearrangement of the long arm of chromosome 8 (8q11-13) affecting PLAG1 in lipoblastoma, when myxoid liposarcoma shows a t(12;16) (q13;p11) translocation [10-13].

Dilley et al. [14] had reviewed 24 patients who presented to a single institution over a 15 year period. Of these 24 patients, only 4 had tumor in the head and neck region. The tumor may present as a focal or diffuse lesion with the former being more common. It has also been mentioned that focal lesions usually do not require further surgery following initial resection. Patients with lipoblastoma may present with various symptoms depending upon its site. Rasmussen et al. [15], mentioned a case of cervical lipoblastoma causing intermittent airway obstruction. Farrugia et al. [16] reported another case of lipoblastoma of the neck which was presented as a rapidly enlarging mass, mimicking cystic hygroma. Eveline Bruyeera [6] reported three cases of head and neck lipoblastoma. The outcome of three patients was favorable after complete surgical resection. K Premkumar [2] recently reported another case of an extensive tumor located over the nape of the neck that causes a restriction of neck movement.

Lipoblastoma exhibits a propensity to invade locally. If not excised early, it may enlarge and infiltrate the various surrounding structures. Various pressure symptoms may occur which can lead to life threatening complications as noted hereinbefore [2].

The mainstay of treatment is complete non-mutilating resection of the tumor to avoid recurrence. If the entire tumor cannot be safely removed at the time of initial resection, a staged approach is recommended. [17] The prognosis is excellent, and the recurrence rate is less than $25 \%$ being largely attributed to the infiltrative form 'lipoblastomatosis'. The evolution is unpredictable, spontaneous resolution and maturation into lipoma may occur, but metastases still not reported so far $[17,18]$.

\section{Conclusion}

Head and neck lipoblastoma are a rare childhood tumor, usually presenting as a progressive painless mass, rarely causing airway obstruction, nerve or vascular compression. Through our case, we aimed to shed light on lipoblastomas as a differential diagnosis of rapidly growing soft fatty masses of children within this region.

\section{References}

1. Chung EB, Enzinger FM (1973) Benign lipoblastomatosis: An analysis of 35 cases. Cancer 32(2): 482-492.
2. Premkumar K, Basle MA, Jassim K, Waseem Ahamed TP (2015) An unusual case of cervical lipoblastoma with review of literature. J Cancer Res Ther 11(4):1025.

3. Jaffe RH (1926) Recurrent lipomatous tumors of the groin: Liposarcoma and lipoma pseudomyxomatodes. Arch Pathol 1: 381-387.

4. Papaioannou G, Sebire N, McHugh K (2009) Imaging of the unusual pediatric blastomas. Cancer Imaging 9: 1-11.

5. Pham NS, Poirier B, Fuller SC, Dublin AB, Tollefson TT (2010) Pediatric lipoblastoma in the head and neck: a systematic review of 48 reported cases. Int J Pediatr Otorhinolaryngol 74(7): 723-728.

6. Bruyeer E, Lemmerling M, Poorten VV, Sciot R, Hermans R (2012) Paediatric lipoblastoma in the head and neck: three cases and review of literature. Cancer Imaging 12: 484-487.

7. Murphey MD, Carroll JF, Flemming DJ, Pope TL, Gannon FH, et al. (2004) From the archive from AFIP. Benign musculoskeletal lipomatous lesions. Radiographics 24(5): 1433-1466.

8. Toirkens J, De Schepper AM, Vanhoenacker F, Van Dyck P, Gielen J, et al. (2011) A comparison between histopathology and findings on magnetic resonance imaging of subcutaneous lipomatous soft-tissue tumors. Insights Imaging 2(5): 599-607.

9. Ohjimi Y, Iwasaki H, Kaneko Y, Ishiguro M, Ohgami A, et al. (1992) A case of lipoblastoma with t (3;8)(q12;q11.2). Cancer Genet Cytogenet 62(1): 103-105.

10. De Saint Aubain Somerhausen N, Coindre JM, Debiec-Rychter M, Delplace J, Sciot R (2008) Lipoblastoma in adolescents and young adults: report of six cases with FISH analysis. Histopathology 52(3): 294-298.

11. Hibbard MK, Kozakewich HP, Dal Cin P, Sciot R, Tan X, et al. (2000) PLAG1 fusion oncogenes in lipoblastoma. Cancer Res 60(17): 48694872.

12. Tallini G, Akerman M, Dal Cin P, De Wever I, Fletcher CD, et al (1996) Combined morphologic and karyotypic study of 28 myxoidliposarcomas. Implications for a revised morphologic typing (a report from the CHAMP Group). Am J Surg Pathol 20(9): 1047-1055.

13. Sciot R, De Wever I, Debiec-Rychter M (2003) Lipoblastoma in a 23-year-old male: distinction from atypical lipomatous tumor using cytogenetic and fluorescence in-situ hybridization analysis. Virchows Arch 442(5): 468-471.

14. Dilley AV, Patel DL, Hicks MJ, Brandt ML (2001) Lipoblastoma: pathophysiology and surgical management. J Pediatr Surg 36(1): 229231.

15. Rasmussen IS, Kirkegaard J, Kaasbol M (1997) Intermittent airway obstruction in a child caused by a cervical lipoblastoma. Acta Anaesthesiol Scand 41(7): 945-946.

16. Farrugia MK, Fearne C (1998) Benign lipoblastoma arising in the neck. Pediatr Surg Int 13(2-3): 213-214.

17. Pederiva F, Zanazzo GA, Gregori M, Schleef J (2013) Suprascapular lipoblastoma extending in to the thorax. APSP J Case Rep 4(2): 20.

18. Kok KY, Telisinghe PU (2010) Lipoblastoma: clinical features, treatment, and outcome. World J Surg 34(7): 1517-1522. 
This work is licensed under Creative Commons Attribution 4.0 License DOI: 10.19080/JHNSS.2018.03.555621 